\title{
Placental transport studied by means of isolated plasma membrane vesicles
}

\author{
BY C. A. R. BOYD \\ Department of Human Anatomy, University of Oxford, South Parks Road, Oxford OX1 $3 Q X$
}

It has recently become possible to isolate relatively easily pure preparations of membranes from the brush border as well as from the basal surface of the term human trophoblast. Such membranes reseal to form closed structures which are called 'vesicles'. Many studies on solute transport have been performed using such vesicles because they permit experimental investigation of transport systems, and of their driving forces and regulation at the level of the membrane transport proteins responsible for membrane solute transport. Such an analysis cannot readily be performed using other experimental methods. In the present article several transport systems present in the human placenta which have been studied by means of vesicle experiments will be reviewed. Attention will also be drawn to the limitations and potential pitfalls of such experiments.

\section{QUESTIONS TO BE ASKED WHEN USING MEMBRANE VESICLES}

As with all scientific analysis the first and most important question to be asked is 'what is the experimental model being used for?' In most instances, studies with vesicles are used to analyse, at the membrane level, physiological phenomena which have been described in more complex systems, for example in vivo or using perfused placentas or in experiments on intact trophoblast in vitro. They also can be used to establish the presence and properties of transport systems which previously have not been studied, although in this case the physiological significance of experimental findings may prove difficult to establish. In this instance the use of vesicle methodology is no different than the use, for example, of enzyme biochemistry to study metabolic processes; indeed transport systems be they carrier-, pump- or channel-mediated may be considered as 'vectorial enzymes' separating two fluid phases and it is the investigation of the properties of such 'enzymes' that is the aim of these experiments. Importantly vesicles also allow the investigation of mechanisms of control of transport, for example by altering the extent of transport protein phosphorylation.

Assuming that the experimental aim has been carefully thought about, more practical problems then need to be considered. The first of these concerns the purity of the membranes isolated. Since the work of Smith et al. (1974) brush-border-membrane vesicles have been readily available using very straightforward biochemical methods (see for example, Kudo et al. 1987; Glazier et al. 1988). More recently C. H. Smith and colleagues (Kelly et al. 1983) have developed a method for the isolation of basal membrane vesicles which has been successfully employed as will be discussed later. Marker enzyme (or receptor) analysis, using alkaline phosphatase (EC 3.1.3.1) for the brush border and adenylate cyclase $(E C 4.6 .1 .1)$ or $\beta$-adrenoreceptors for basal membranes, shows that such preparations are properly enriched usually by a factor of about 30 over the starting homogenate. Cross contamination appears not to be an appreciable problem since there is negative enrichment of the marker enzymes for the contralateral membrane. 
The second practical consideration concerns the orientation of such isolated vesicles; this can be assessed by several methods but the use of detergents to lyse the vesicles and, thus, reveal latent enzyme activity is probably the most widely used. It is based on the known topology of membrane-bound enzyme activity with respect to the location of the active site. For example, alkaline phosphatase is a membrane-bound ectoenzyme with substrate hydrolysis occurring at the external face of the plasma membrane in the intact tissue. The anionic substrates for alkaline phosphatase (such as the nitrophenylphosphates) are impermeant and, therefore, will only be hydrolysed by vesicles that retain their anatomically correct orientation (right-side-out) but will not be hydrolysed by vesicles which are inside-out. However, vesicle lysis by detergent will allow substrate to gain access to the enzyme in such inside-out vesicles and the ratio of this increment in activity on lysis ('latent activity'): total activity is directly proportional to the fraction of vesicles which are inside-out. It turns out that brush-border-membrane vesicles are overwhelmingly right-side-out. For basal vesicles our state of knowledge is less clear but unpublished work by Marin and his coworkers quoted by El Mir (1990) suggests that these vesicles may not be orientated in such a uniform manner.

When conducting transport experiments vesicles are incubated with substrate, usually radioactively labelled, and portions removed so as to determine the quantity of substrate which has become associated with the vesicle. This is usually described as 'uptake' of substrate and can only be determined if the vesicles are successfully separated from the medium in which they have been incubated. How may such separation be achieved? The most popular method depends on filtration in which the portion is rapidly pulled by vacuum through a filter (containing small-diameter pores, sufficiently small so as to impede vesicle filtration, but large enough to permit rapid removal of the incubation medium when the vacuum is applied). The use of filters of different composition and of different pore size may result in differences between different laboratories because, for example, smaller vesicles may not be retained by the filter; one way of testing the extent of vesicle retention is to measure the recovery of marker enzyme both on the filter itself and in the filtrate. Separation of vesicles may also be successfully achieved by other methods (Shennan \& Boyd, 1987).

How may one distinguish the transport of solute from binding to the external surface of the vesicles? The classical way (see Murer \& Hopfer, 1980) of distinguishing binding from transport is to vary the osmolarity of the incubation medium so as to shrink or swell the vesicles, thus altering their intravesicular volume; at equilibrium this will alter the quantity of solute within the vesicle but will be without effect on binding since the area of membrane surface is not altered. An alternative experimental procedure is to lyse the vesicles with low concentrations of detergent so as to abolish transport but to retain binding. A combination of both methods, particularly if they give concordant results, is often reassuring; it is important that in any novel investigation using a different solute the extent to which uptake is determined by binding or transport is measured.

\section{RESULTS OF EXPERIMENTS ILLUSTRATING METHODOLOGICAL PROBLEMS}

Sulphate transport across the placental brush-border membrane has been investigated extensively (Boyd \& Shennan, 1986a,b) and the experimental evidence shows rather clearly that the process is electroneutral and carrier-mediated. Some of the evidence for this is illustrated in Fig. 1. The uptake of sulphate is time-dependent and approaches 

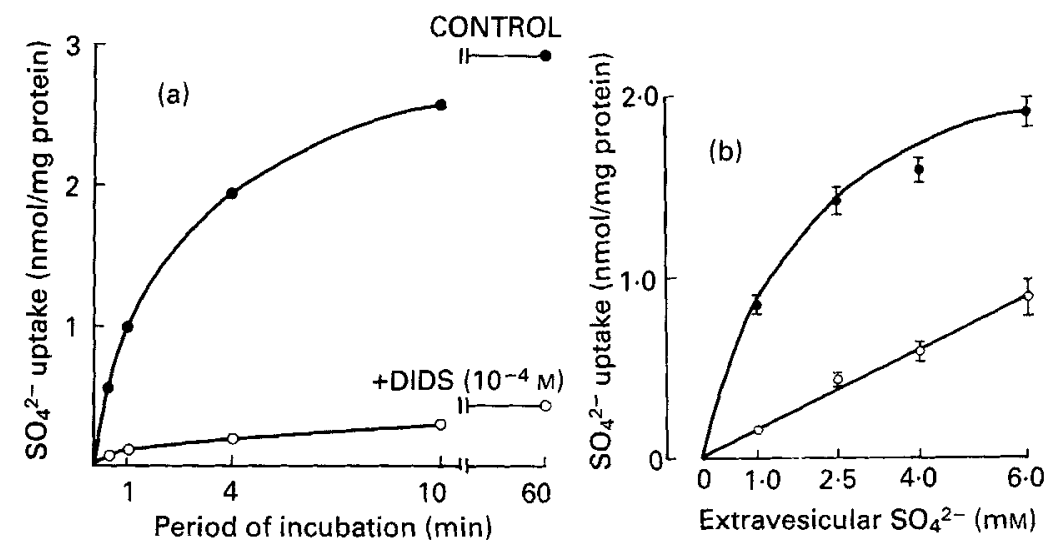

(c)

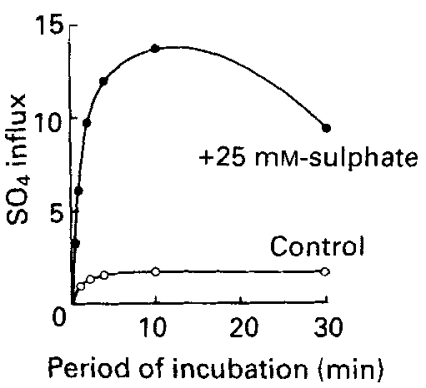

Fig. 1. (a) Inhibition of sulphate influx by DIDS $\left(10^{-4} \mathrm{M}\right.$ ) (from Boyd \& Shennan, 1986a). (b) Sulphate influx as a function of medium sulphate concentration with $(O)$ or without $(O) 10^{-4} \mathrm{M}-\mathrm{DIDS}$ (from Boyd \& Shennan, $1986 a$ ). (c) Trans stimulation of sulphate influx by preloading of vesicles with (O) or without (O) 25 mM-sodium sulphate (from Bustamente et al. 1988).

equilibrium within $10 \mathrm{~min}$; it is specifically inhibited by low concentrations of the wellknown anion exchange inhibitor, 4,4'-diisothiocyanatostilbene-2,2' disulphonic acid (DIDS; Fig. 1(a)); it shows saturation with higher concentrations of substrate (Fig. 1(b)); and it exhibits counter transport so that influx of labelled sulphate is very markedly stimulated when the vesicles are preloaded with unlabelled substrate (Fig. 1(c)). All three of these properties are characteristic of carrier-mediated transporters. Moreover, sulphate transport is electroneutral since it is not altered by imposition of a diffusion potential using potassium ion gradients and the $\mathrm{K}$ ionophore valinomycin (Fig. 2(a)) These experiments show the strengths of vesicle analysis in the study of transport of this particular anion (incidentally the transporter involved also translocates the nutritionally important oxyanions selenate, molybdate, tungstate and chromate; Boyd \& Shennan, $1986 b$; Shennan, 1988).

In contrast to the lack of effect of an imposed electrical potential on sulphate movement, in the case of chloride ions (Fig. 2(b)) a diffusion potential (inside positive) stimulates chloride entry through a DIDS-insensitive system, presumably a chloride conductance. Interestingly brush-border-membrane vesicles also show chloride transport via anion exchange (Shennan et al. 1986).

Recent work on amino acid transport into basal membrane vesicles has been carried out by Smith and colleagues (for example, see Hoetzli \& Smith, 1989). We have also investigated amino acid transport in basal vesicles with particular reference to the differences in amino acid transporters found at the apical and basal surfaces of the trophoblast (Kudo \& Boyd, 1990a). We have studied tyrosine transport in both membranes under identical experimental conditions (Kudo \& Boyd, 1990b). As shown in Fig. 3(a), the osmotic sensitivity of tyrosine uptake is demonstrated in both vesicle preparations; the detergent (Triton X-100) sensitivity of such uptake is also shown and confirms that transport is the major component, with binding accounting only for about $15 \%$ of uptake even for this relatively hydrophobic amino acid. The transport systems present in the apical and basal surfaces of the vesicles are clearly quite different for this 

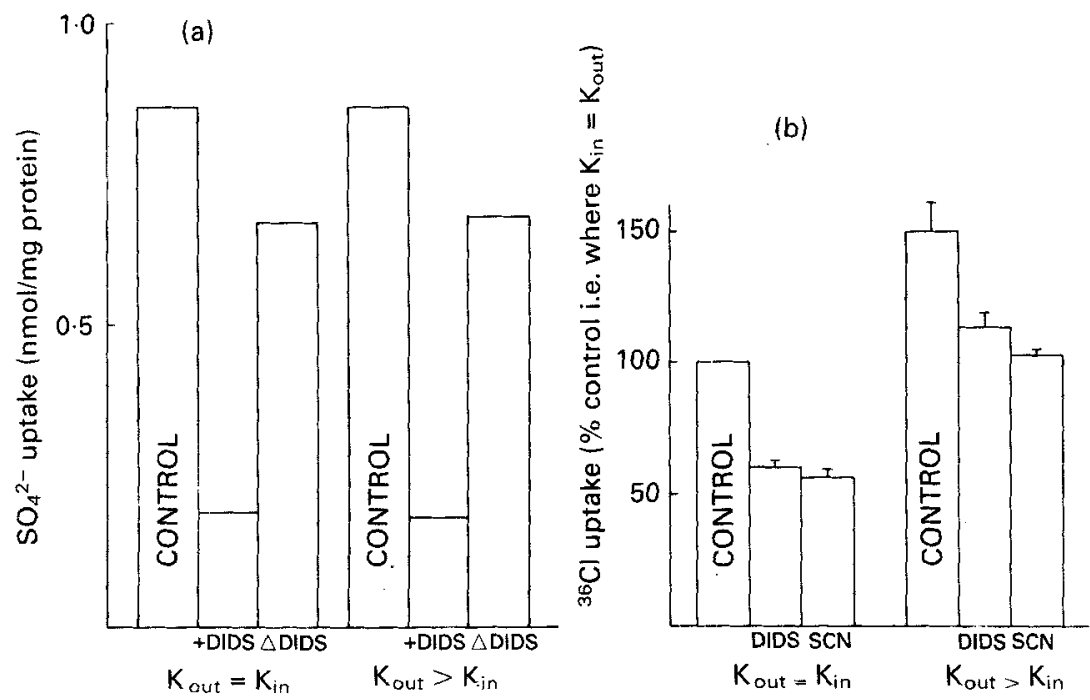

Fig. 2. (a) Electroneutrality of sulphate transport as shown by the absence of stimulation by an inside positive membrane potential (induced by a potassium diffusion potential) as compared with membrane vesicles voltage clamped to zero potential. Valinomycin $(20 \mu \mathrm{g} / \mathrm{mg}$ protein) was used to increase $\mathrm{K}$ permeability in the presence of the $\mathrm{K}$ gradients indicated. DIDS, where indicated, was added at $10^{-4} \mathrm{M}$ (from Boyd \& Shennan, 1986a). $\triangle D I D S$ shows the difference in uptake between systems with and without inhibitor. (b) Voltage dependence of chloride transport into placental brush-border-membrane vesicles in the presence or absence of a $\mathrm{K}$ diffusion potential (from Shennan et al. 1986). Note stimulation of chloride influx by an inside positive potential and that this stimulation is confined to the DIDS-intensive fraction of chloride transport. Thiocyanate (SCN) (10 mM) inhibits the same moiety of chloride fiux as does DIDS (i.e. the electroneutral anion-exchange flux).

Table 1. Effect of amino acids on L-tyrosine $(10 \mu \mathrm{M})$ uptake by membrane vesicles (after Kudo \& Boyd, 1990b)

(Mean values with their standard errors)

\begin{tabular}{|c|c|c|c|c|}
\hline \multirow[b]{3}{*}{ Inhibitor $(10 \mathrm{mM})$} & \multicolumn{4}{|c|}{ Percentage of control uptake } \\
\hline & \multicolumn{2}{|c|}{ Brush-border vesicles } & \multicolumn{2}{|c|}{ Basal vesicles } \\
\hline & Mean & SE & Mean & $\mathrm{SE}$ \\
\hline Nil & \multicolumn{2}{|c|}{100} & \multicolumn{2}{|c|}{100} \\
\hline L-tyrosine & $10 \cdot 2$ & 1.9 & $55 \cdot 5$ & $1 \cdot 3$ \\
\hline D-tyrosine & $43 \cdot 4$ & $3 \cdot 6$ & $66 \cdot 1$ & $1 \cdot 6$ \\
\hline $\mathrm{BCH}$ & $9 \cdot 8$ & $0 \cdot 6$ & $71 \cdot 2$ & $6 \cdot 3$ \\
\hline Methyl AIB & $99 \cdot 1$ & $4 \cdot 6$ & $92 \cdot 3$ & $7 \cdot 2$ \\
\hline
\end{tabular}

$\mathrm{BCH}$, bicycloheptane (leucine transport system substrate); Methyl AIB, methyl aminoisobutyric acid (alanine transport system substrate).

particular amino acid (Table 1), although neither is sodium-dependent. This exemplifies a general property of the placenta with respect to handling of amino-nitrogen, where asymmetry of transport is a pronounced feature of the cellular functioning of the trophoblast. It is possible to perform kinetic studies on vesicles and Fig. 3(b) shows very 
(a) Effect of medium osmolarity on tyrosine uptake
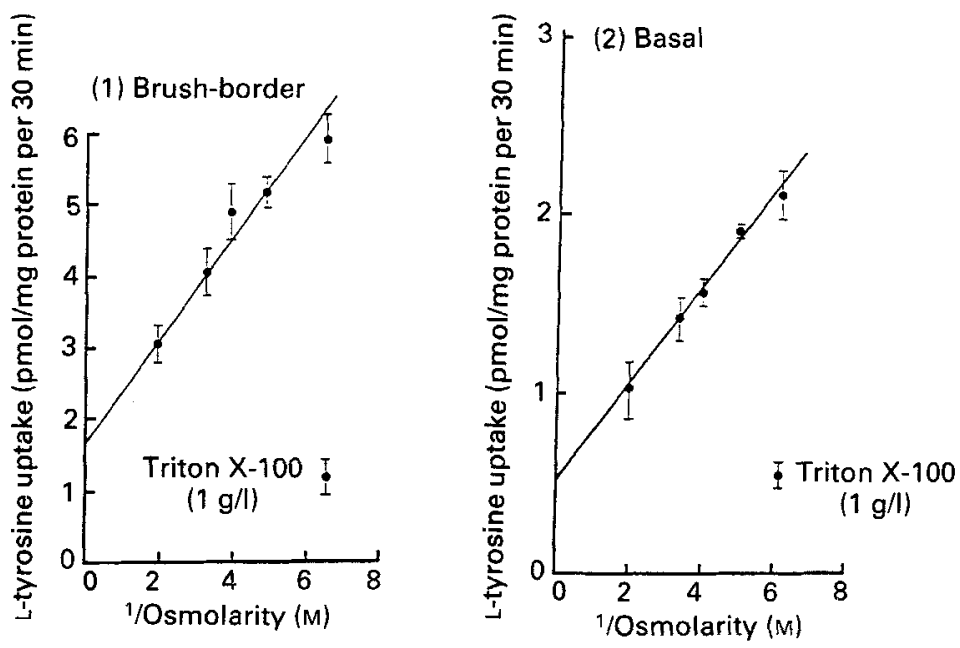

(b) Phenylalanine inhibition of tyrosine uptake
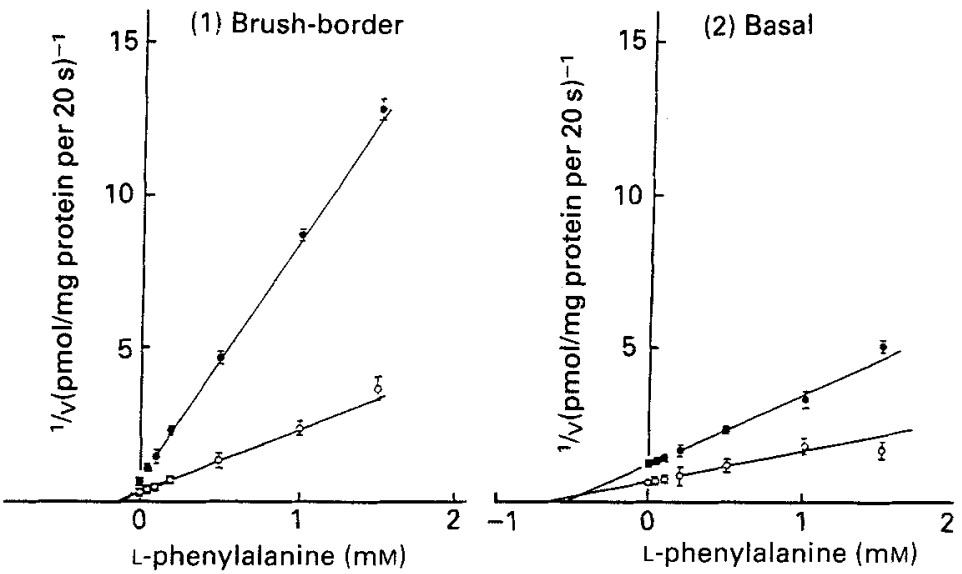

Fig. 3. Osmotic sensitivity of tyrosine infiux into vesicles from (1) apical or (2) basal surfaces of the term human placenta (from Kudo \& Boyd, 1990b). (b) Kinetics of inhibition by phenylalanine of tyrosine influx into vesicles prepared from apical (1) or basal (2) plasma membranes of term human placenta. (O), $2 \mu \mathrm{M}$-tyrosine; (๑), $5 \mu$ M-tyrosine (from Kudo \& Boyd, 1990b). Points are means with their standard errors represented by vertical bars.

clearly, using a Dixon plot (a standard enzymological kinetic analysis), that phenylalanine competes with tyrosine at both surfaces of the placenta but does so with quite different affinities.

\section{FUTURE PROSPECTS FOR VESICLE STUDIES ON THE PLACENTA}

The value of efflux studies is only just beginning to be appreciated but they clearly represent an important method for studying transport in vesicles since the external 
medium may be altered very readily permitting investigation of the interactions between solute at the internal and external faces of the transporter (see Deves \& Boyd, 1989). A recent example of this is given in the work of Marin et al. (1990) who have shown that bile acid transport is via an electroneutral exchanger in vesicles prepared from the basal surface of the trophoblast and that bicarbonate gradients will power taurocholate movement across this face of the trophoblast.

Another area ripe for further investigation is the development of methods for measuring non-isotopic solute flux; recent studies (Kudo et al. 1989) show that high-performance liquid chromatography can be used to determine vesicle antibiotic content so that transport can be measured if sufficient sensitivity of the analytical procedure is available.

It seems likely that future placental studies will focus in particular on regulatory aspects of vesicle transport, particularly with reference to short-term regulation of membrane-transport protein function. Additionally vesicles may well be used to investigate transport using alternative assay systems, for example in doped lipid bilayers, so as to measure the activity of single-channel currents. Additionally vesicles will be required for the functional assay of membrane transport proteins during their purification and in this context the placenta represents a wonderful potential source of human membrane transport proteins as well as of their mRNA.

I am grateful to my colleagues for experimental work and to the MRC for financial support.

\section{REFERENCES}

Boyd, C. A. R. \& Shennan, D. B. (1986a). Human placental sulphate transport: studies on chorionic trophoblast brush border membrane vesicles. Journal of Physiology 377, 15-24.

Boyd, C. A. R. \& Shennan, D. B. (1986b). Sulphate transport into vesicles prepared from human placenta: inhibition by trace element oxides. Journal of Physiology 379, 367-376.

Bustamente, C., Yudilevich, D. L. \& Boyd, C. A. R. (1988). A new form of asymmetry in epithelia: kinetics of apical and basal sulphate transport in human placenta. Quarterly Journal of Experimental Physiology 73, 1013-1016.

Deves, R. \& Boyd, C. A. R. (1989). The determination of kinetic parameters for carrier mediated transport of non-labelled substrate analogues. Proceedings of the Royal Society of London B 237, 85-97.

El Mir, M.-Y. A.-K. (1990). Transport of taurocholate across the placental basal membrane. PhD Thesis, University of Salamanca.

Glazier, J. D., Jones, C. J. P. \& Sibley, C. P. (1988). Transport of sodium into vesicles prepared by three different methods. Biochimica Biophysica Acta 945, 127-134.

Hoetzli, S. D. \& Smith, C. H. (1989). Alanine transport systems in isolated basal plasma membrane of human placenta. American Journal of Physiology 256, C630-C637.

Kelly, L. K., Smith, C. H. \& King, B. F. (1983). Isolation and partial characterization of the basal cell membrane of the human placental trophoblast. Biochimica Biophysica Acta 734, 91-98.

Kudo, Y. \& Boyd, C. A. R. (1990a). Characterization of amino acid transport systems in human placental basal membrane vesicles. Biochimica Biophysica Acta 1021, 169-174.

Kudo, Y. \& Boyd, C. A. R. (1990b). Human placental L-tyrosine transport: a comparison of brush border and basal membrane vesicles. Journal of Physiology 426, 381-395.

Kudo, Y., Urabe, T., Fujiwara, A., Yamada, K. \& Kawasaki, T. (1989). Cephalexin transport into placental membrane vesicles. Biochimica Biophysica Acta 978, 313-318.

Kudo, Y., Yamada, K., Fujiwara, A. \& Kawasaki, T. (1987). Characterization of amino acid transport systems in human placental brush border membrane vesicles. Biochimica Biophysica Acta 904, 309-319.

Marin, J. J. G., El Mir, M.-Y. A.-K., Eleno, N., Serrano, M. A. \& Boyd, C. A. R. (1990). Taurocholate transport in human placental basal membrane vesicles. Gastroenterology 99, 1431-1438. 
Murer, H. \& Hopfer, U. (1980). Transport studies using isolated membrane vesicles. Journal of Membrane Biology 58, 100-118.

Shennan, D. B. (1988). Selenate transport by human placental microvillus membrane vesicles. British Journal of Nutrition 59, 13-19.

Shennan, D. B. \& Boyd, C. A. R. (1987). Ion transport by the placenta: a review of membrane transport systems. Biochimica Biophysica Acta 906, 437-457.

Shennan, D. B., Davis, B. \& Boyd, C. A. R. (1986). Chloride transport in human placental microvillus membrane vesicles. European Journal of Physiology 406, 60-64.

Smith, N. C., Brush, M. G. \& Luckett, S. (1974). Preparation of human placental villous surface membrane. Nature 252, 302-303. 